\title{
Studies on Cyclic Ketones: Synthetic Routes to Indenopyridine, Indenopyran, Fluoreneoxime, Pyrazoloindenotriazine and Indenopyridazine Derivatives
}

F.M.A. El-Taweel" and G . Zaied

Department of Chemistry, Faculty of Science, (Damietta) New

Damietta, Egypt.

\begin{abstract}
SEVERAL new indenopyridine, indenopyran, fluorenoneoxime, Sindenopyridazine and pyroloindenotriazine derivatives were prepared using cyclic ketones (1), arylmethylenenitriles (2) and active methylene nitriles 3 as starting materials.
\end{abstract}

Cyclic ketons are versatile reagents, which have been extensively utilized for synthesis of functionally substituted aromatic and heteroaromatic systems ${ }^{(1-9)}$. These aromatic and heteroaromatic systems are interesting as potential biodegrable agrochemicals ${ }^{(1)}$, pharmaceuticals ${ }^{(3,4)}$, rodenticides ${ }^{(10)}$ and blood anticoagulants $^{(5)}$. The present work has resulted in the formation of several new indenopyridine, indenopyran, fluoreneoxime, indenopyridazine and pyrazolo $[3,2-c]$ indeno $[1,2-e][1,2,4]$ triazine derivatives of potential biological importance using cyclic ketones (1), arylmethylenenitriles (2) and active methylene nitriles (3) as starting materials.

It has been found that, cyclic ketones $1 \mathrm{~b}, \mathrm{~d}$ reacted readily with arylmethylenenitriles (2) in ethanol containing piperidine to yield (1:1) adducts. Thus, the indeno $[1,2-b]$ pyrans (5) or the indeno[1,2-b] pyridines (7) can be considered as reaction products. Acyclic structures 4 were readily eliminated by ${ }^{1} \mathrm{H}-\mathrm{NMR}$ spectra which revealed signals at $\delta \approx 4.1-5.0 \mathrm{ppm}$ for one proton linked to $\mathrm{sp}^{3}$ carbon. The indeno $[1,2-b]$ pyridines (7) (which can exist with their tautomeric structures (6) were established as reaction products based on IR spectra which revealed the presence of carbonyl groups at $\tilde{v} \approx 1713-1717 \mathrm{~cm}^{-1}$. Signals at similar positions for similar systems was previously observed ${ }^{(4,5)}$. The formation of indeno [1,2-b] pyridines (7) were assumed to proceed via Michael type addition of the active methylene group in 1 to the activated double bond in 2 to give the Michael adducts 4, which readily cyclized to yield 7 (c.f. Scheme 1).

Similarly, cyclic ketones (1) reacting with 2-(2-oxoindolin-3ylidene)malononitrile (8a), (Z)- ethyl 2-cyano-2-(2-oxoindolin-3-ylidene) acetate (8b) in ethanol containing piperidine as a catalyst afforded spiroanellated indeno $[1,2-b]$ pyridines (10) rather than indeno $[1,2-b]$ pyrans (11) as established by IR spectra which revealed carbonyl group at $\tilde{v} \approx 1700-1715 \mathrm{~cm}^{-1}$.

*E.mail : fathyeltaweel@yahoo.com 
Also, cyclic ketones (1) reacted with ethoxymethylenemalononitrile (12) using the same previous conditions to yield the indeno $[1,2-b]$ pyridines 14 . Compounds 14 were most likely formed via the sequence demonstrated in Scheme 2.

On the other hand, indane-1,3-dione (1a) underwent condensation with aromatic aldehydes to give 2-(arylmethylene)-1H-indene-1,3-(2H)-diones $(15)^{(11)}$. Michael condensation of compounds 15 with active methylene nitrile $3 \mathrm{c}^{(12)}$ in ethanol and in the presence of piperidine afforded indeno[1,2-b]pyrans (17). The formation of 17 finds support from correct analytical, spectral data and independent synthesis through the Michael condensation of 1 with the arylmethylenenitriles $(18)^{(12)}$ in ethanol / piperidine (c.f. Scheme 3$)$.

Indanylidenepropanedinitriles (19) were prepared by condensation of cyclic ketones (1) with malononitrile in dry benzene containing catalytic amount of ammonium acetate and glacial acetic acid using water separator, by refluxing for $6 \mathrm{hr}$. Compound 19a reacted with arylmethylenenitriles (2) in refluxing ethanol / piperidine to yield the e fluoreneoximes (23) rather than indeno [1,2- $b$ ] pyridines (21). Structurs 23 were established as reaction products based on their elemental analysis and spectral data. Compounds 23 were assumed to be formed via addition of acidic $\mathrm{CH}$ group in 19a to the activated double bond in the arylmethylenenitriles (2) to give the intermediates 20 which cyclized to 22 and finally aromatized to 23 via hydrogen cyanide or ethyl formate eliminationas recently reported for the formation of similar systems. ${ }^{(3-5)}$

In a similar manner, the ylidenenitrile (19a) was subjected to react with1phenyl-2-nitroethene (24) to give the fluoreneoxime derivative (26). Formation of 26 was assumed to proceed via addition of acidic CH group in 19a to the $\pi$ deficient double in 24 to give the intermediate 25 followed by cyclization and aromatization to yield 26 (c.f. Scheme 4).

The reactivity of cyclic ketones (1) towards aryl diazonium salts and heteroaryl diazonium salts was also studied. Thus, compound 1 was coupled with the aryl diazonium salt to give 1,5-dimethyl-4(2-(1-oxo-1 $H$-inden-2(3H)-ylidene) hyrazinyl-2- phenyl-1H-pyrazol-3-(2H)-ones (27). The later condensed with malononitrile in glacial acetic acid containing an equivalent amount of ammonium acetate under reflux to yield 2-(1,5-dimethyl-3-oxo-2-phenyl-2,3-dihydro-1Hpyrazol-4-yl)-3-oxo-3,9-dihydro- $2 \mathrm{H}$-indeno [2,1-c] pyridazine-4-carbonitriles (28). Compounds 28 were also prepared via reacting the ylidenenitrile 19 with aryl diazonium salt to give 29 , followed by cyclization to 2-(1,5- dimethyl-3- oxo-2phenyl-2,3- dihydro- $1 H$ - pyrazol-4-yl)- 3-oxo-3,9- dihydro- $2 H$ - indeno [2,1-c] pyridazine-4-carbonitriles (28) by the effect of acetic acid.

The heterocyclic diazonium chlorides (30) coupled with cyclic ketones $(1 \mathrm{~b}, \mathrm{c})$ to afford $6 H$-indeno [1,2-e] pyrazolo [5,1-c] [1,2,4] triazin-6-ones (32). Compounds 32 were assumed to be formed via the intermediates (31) which readily underwent cyclization by the reaction of the highly nucleophilic pyrazole $\mathrm{NH}$ (c.f. Scheme 5).

Egypt. J. Chem. 55, No.2 (2012) 

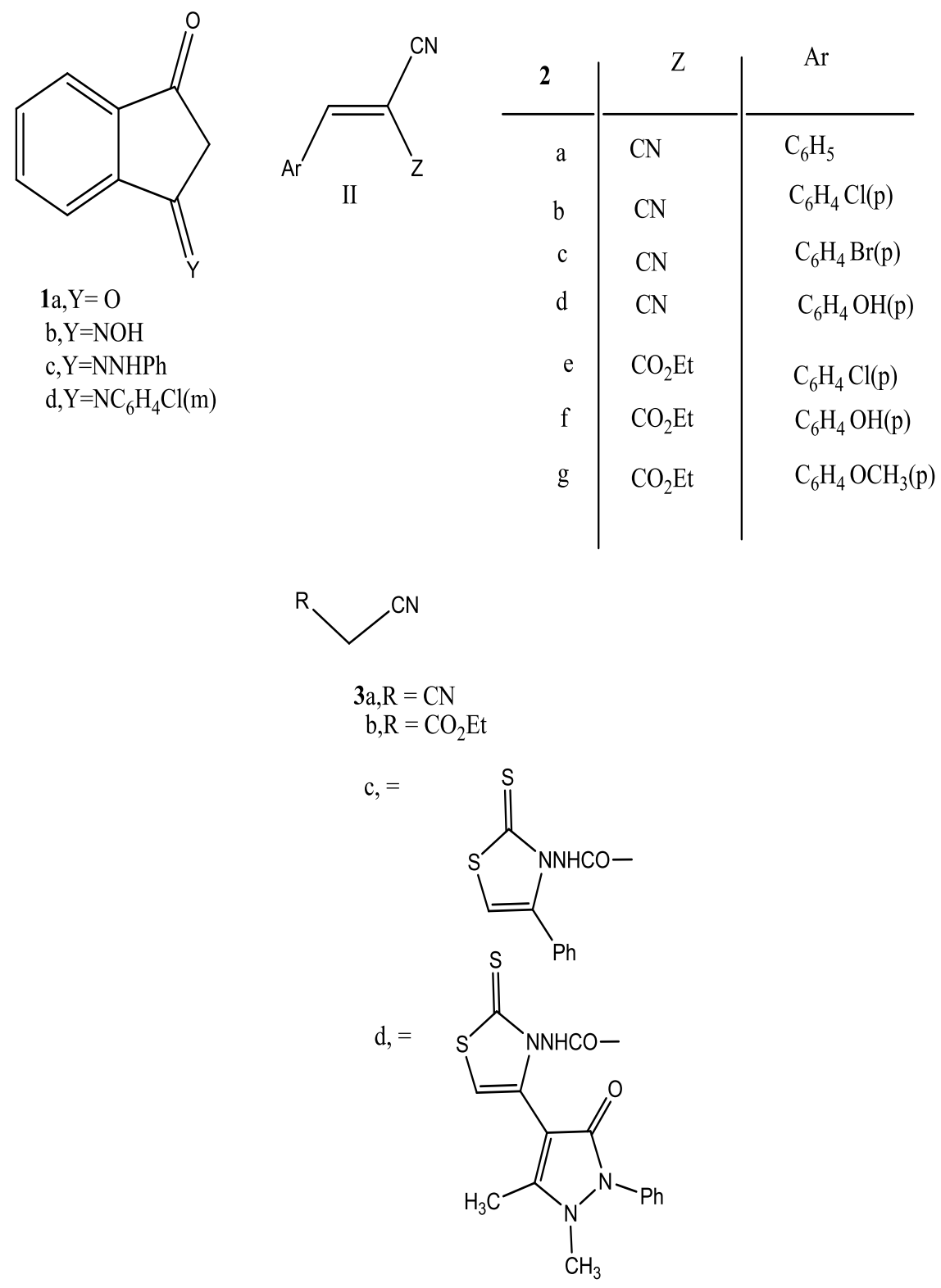

Egypt. J. Chem. 55, No.2 (2012) 

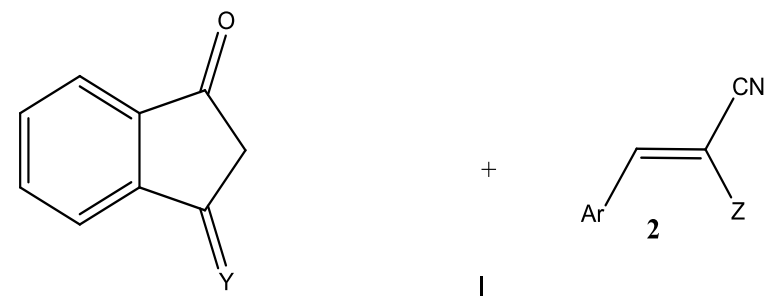

$1 \mathrm{a},=\mathrm{NOH}$

$\mathrm{b},=\mathrm{NC} 6 \mathrm{H} 4 \mathrm{Cl}(\mathrm{m})$
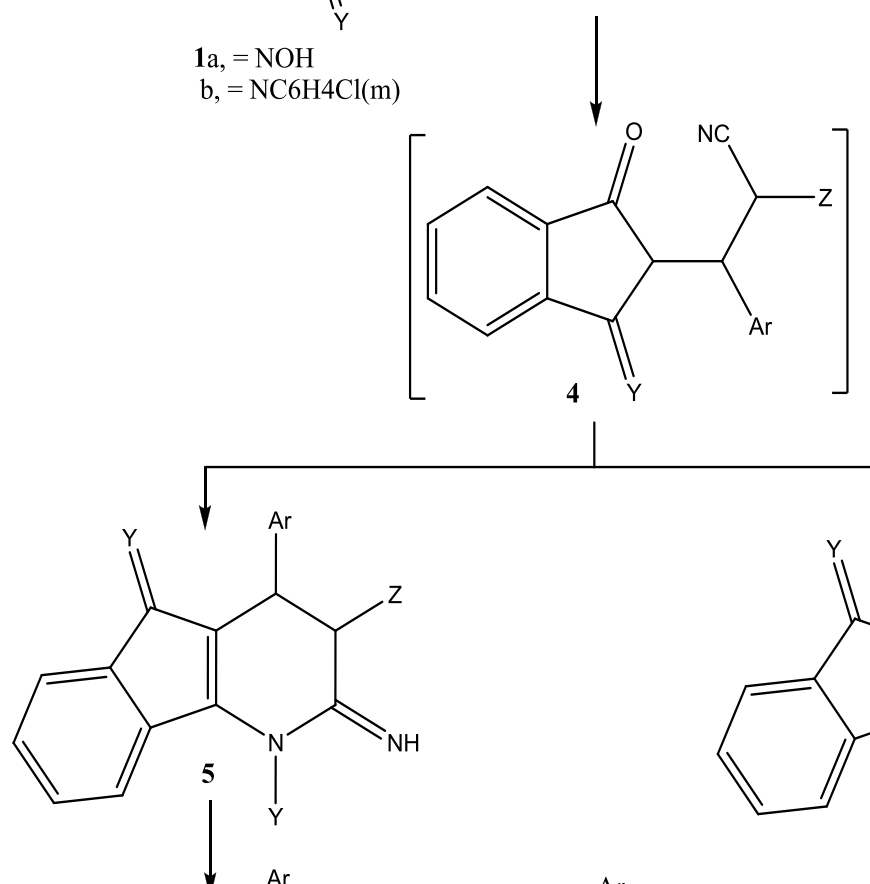<smiles></smiles>

$7 \mathrm{Ar}$

Z Y

a $\quad \mathrm{C}_{6} \mathrm{H}_{5}$

$\mathrm{CN} \quad \mathrm{OH}$

b $\quad \mathrm{C}_{6} \mathrm{H}_{4} \mathrm{Cl}(\mathrm{P})$

$\mathrm{CN} \quad \mathrm{OH}$

c $\quad \mathrm{C}_{6} \mathrm{H}_{4} \mathrm{Br}(\mathrm{P})$

$\mathrm{CN}$

$\mathrm{C}_{6} \mathrm{H}_{4} \mathrm{Cl}(\mathrm{m})$

d $\quad \mathrm{C}_{6} \mathrm{H}_{4} \mathrm{OH}(\mathrm{P}) \quad \mathrm{CN}$

e $\quad \mathrm{C}_{6} \mathrm{H}_{4} \mathrm{OHl}(\mathrm{P}) \quad \mathrm{CO}_{2} \mathrm{Et} \quad \mathrm{OH}$

f $\quad \mathrm{C}_{6} \mathrm{H}_{4} \mathrm{OCH}_{3}(\mathrm{P}) \quad \mathrm{CO}_{2} \mathrm{Et} \quad \mathrm{OH}$

\section{Scheme 1}

Egypt. J. Chem. 55, No.2 (2012) 


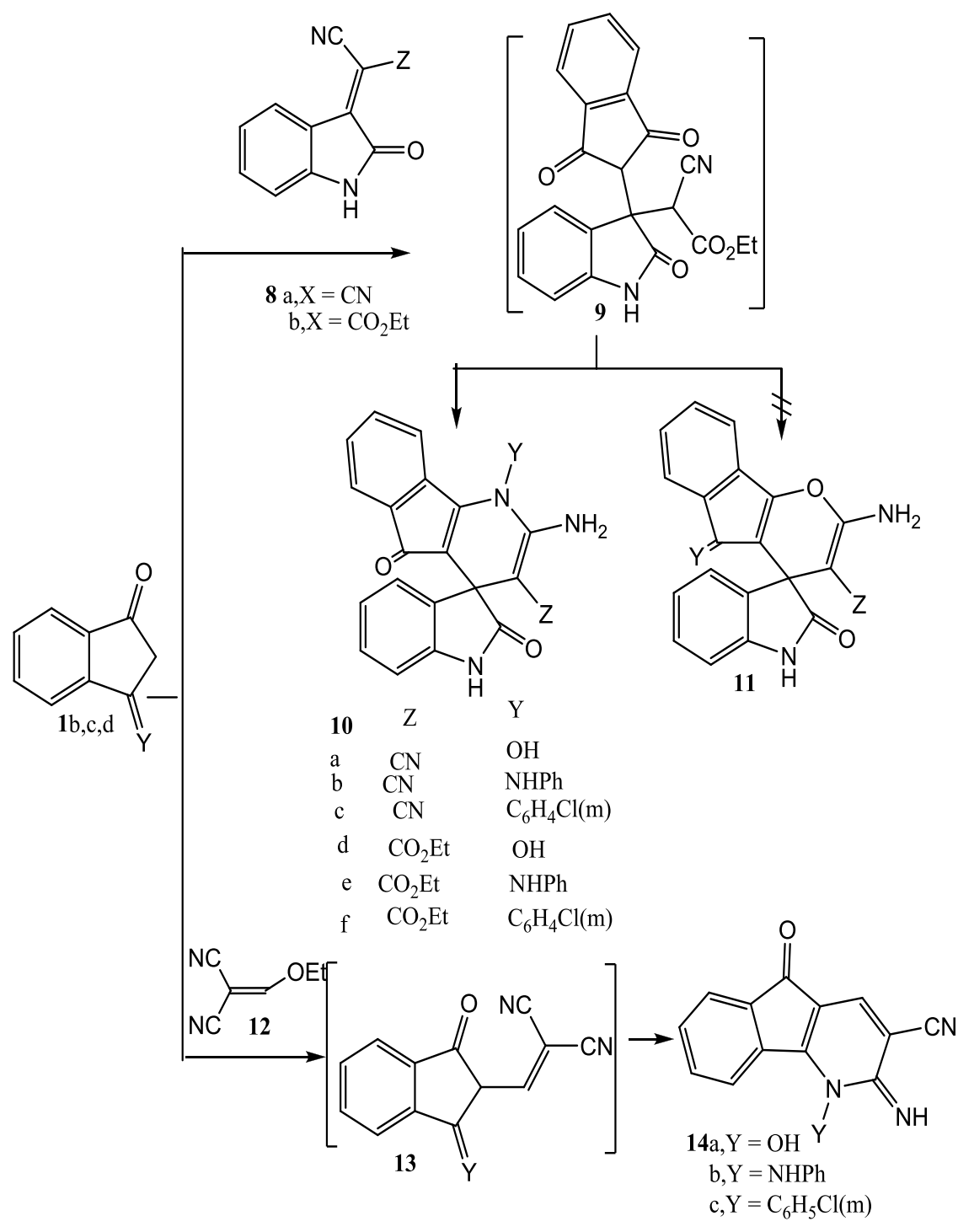

Scheme 2 


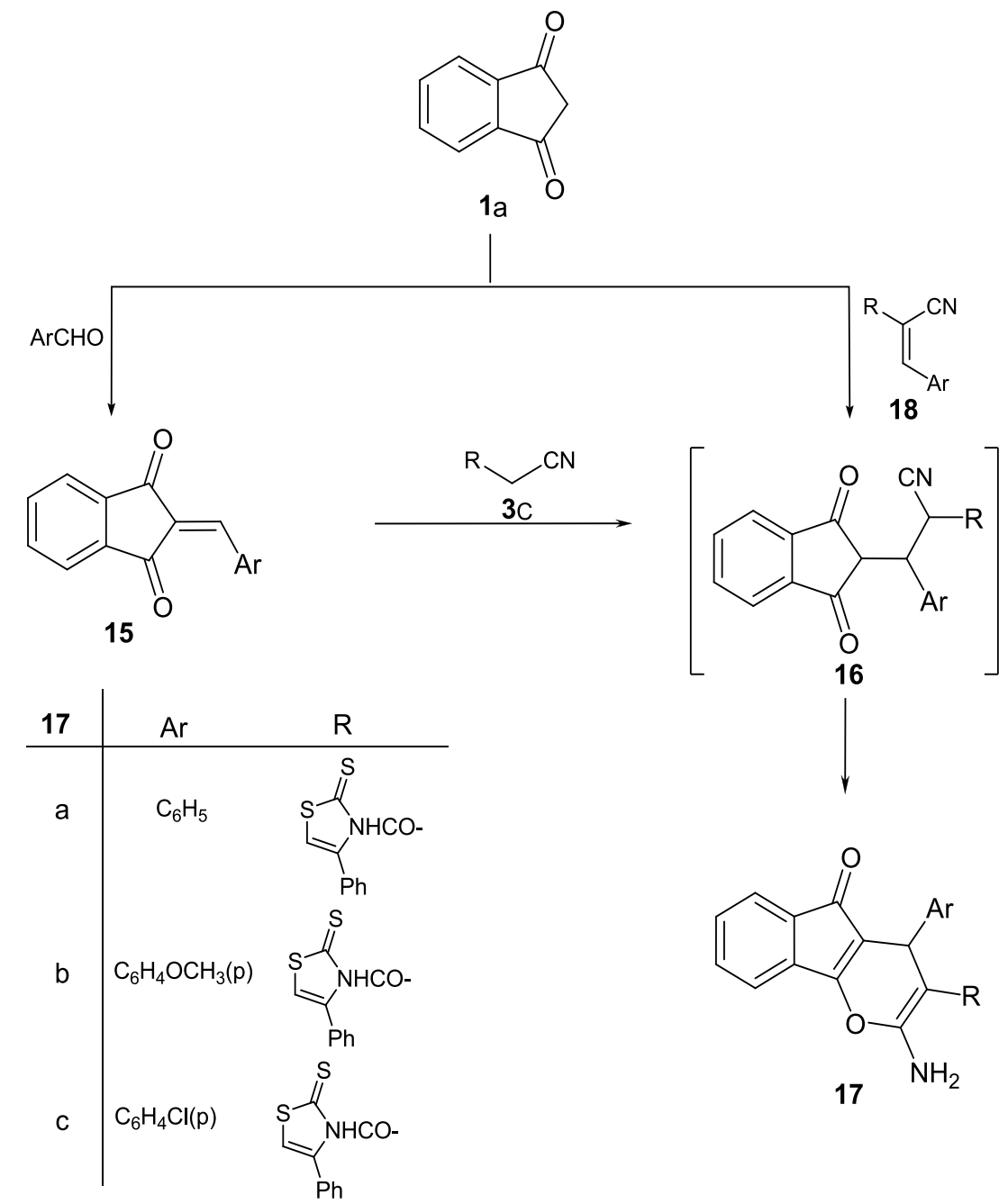

Scheme 3

Egypt. J. Chem. 55, No.2 (2012) 

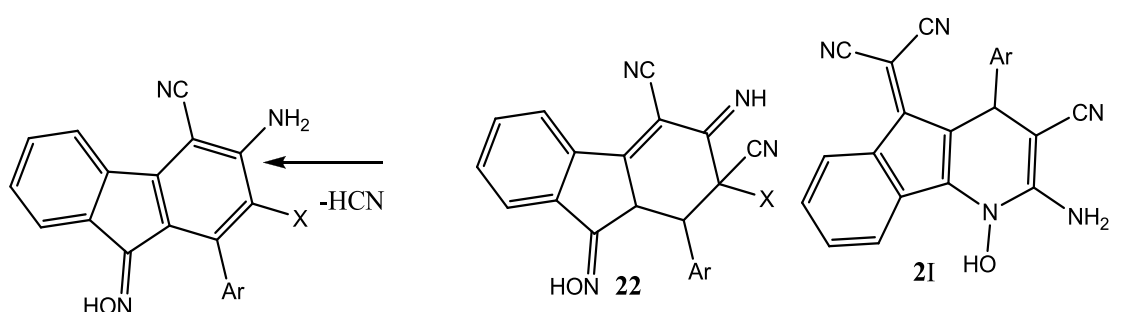

\begin{tabular}{l|l|l}
$\mathbf{2 3}$ & \multicolumn{1}{|c|}{$\mathrm{Ar}$} & \multicolumn{1}{|}{$\mathrm{X}$} \\
\hline $\mathrm{a}$ & $\mathrm{C}_{6} \mathrm{H}_{5}$ & $\mathrm{CN}$ \\
$\mathrm{b}$ & $\mathrm{C}_{6} \mathrm{H}_{4} \mathrm{Cl}(\mathrm{p})$ & $\mathrm{CN}$ \\
c & $\mathrm{C}_{6} \mathrm{H}_{4} \mathrm{Cl}(\mathrm{p})$ & $\mathrm{CO}_{2} \mathrm{Et}$ \\
$\mathrm{d}$ & $\mathrm{C}_{6} \mathrm{H}_{4} \mathrm{OH}(\mathrm{p})$ & $\mathrm{CO}_{2} \mathrm{Et}$
\end{tabular}
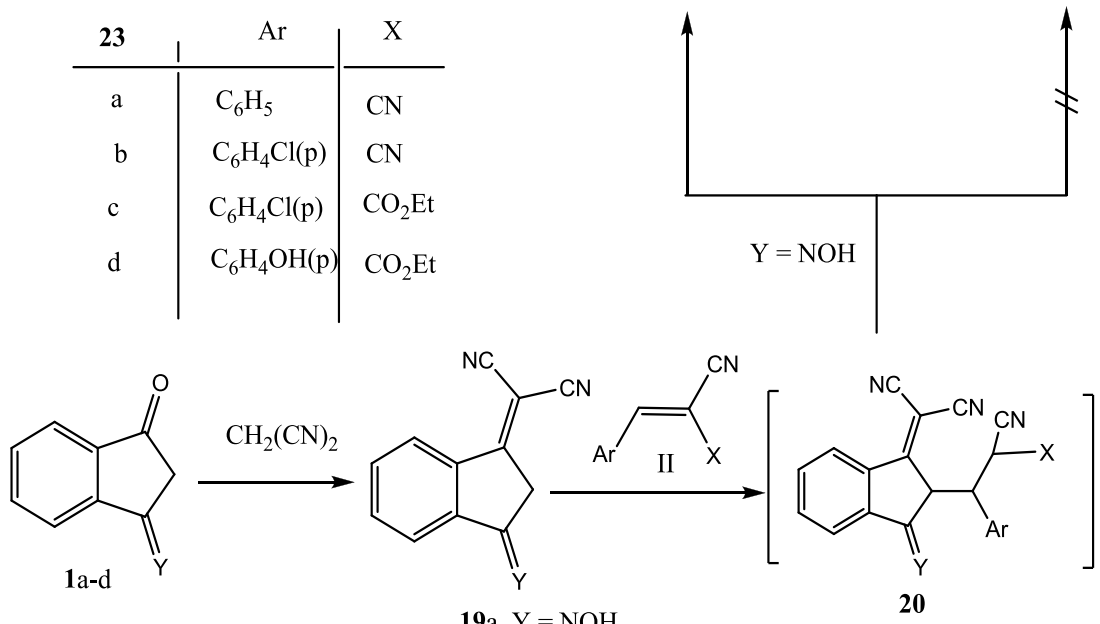

b, $\mathrm{Y}=\mathrm{NNHPh}$
c, $\mathrm{Y}=\mathrm{NC}_{6} \mathrm{H}_{4} \mathrm{Cl}(\mathrm{m})$

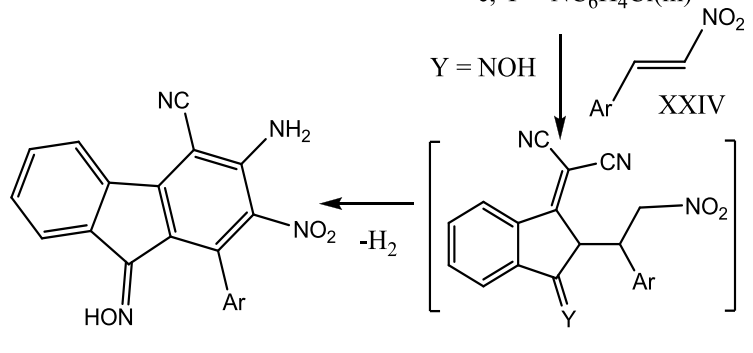

$26 \mathrm{Ar}, \quad \mathrm{C}_{6} \mathrm{H}_{5}$

25

Scheme 4

Egypt. J. Chem. 55, No.2 (2012) 

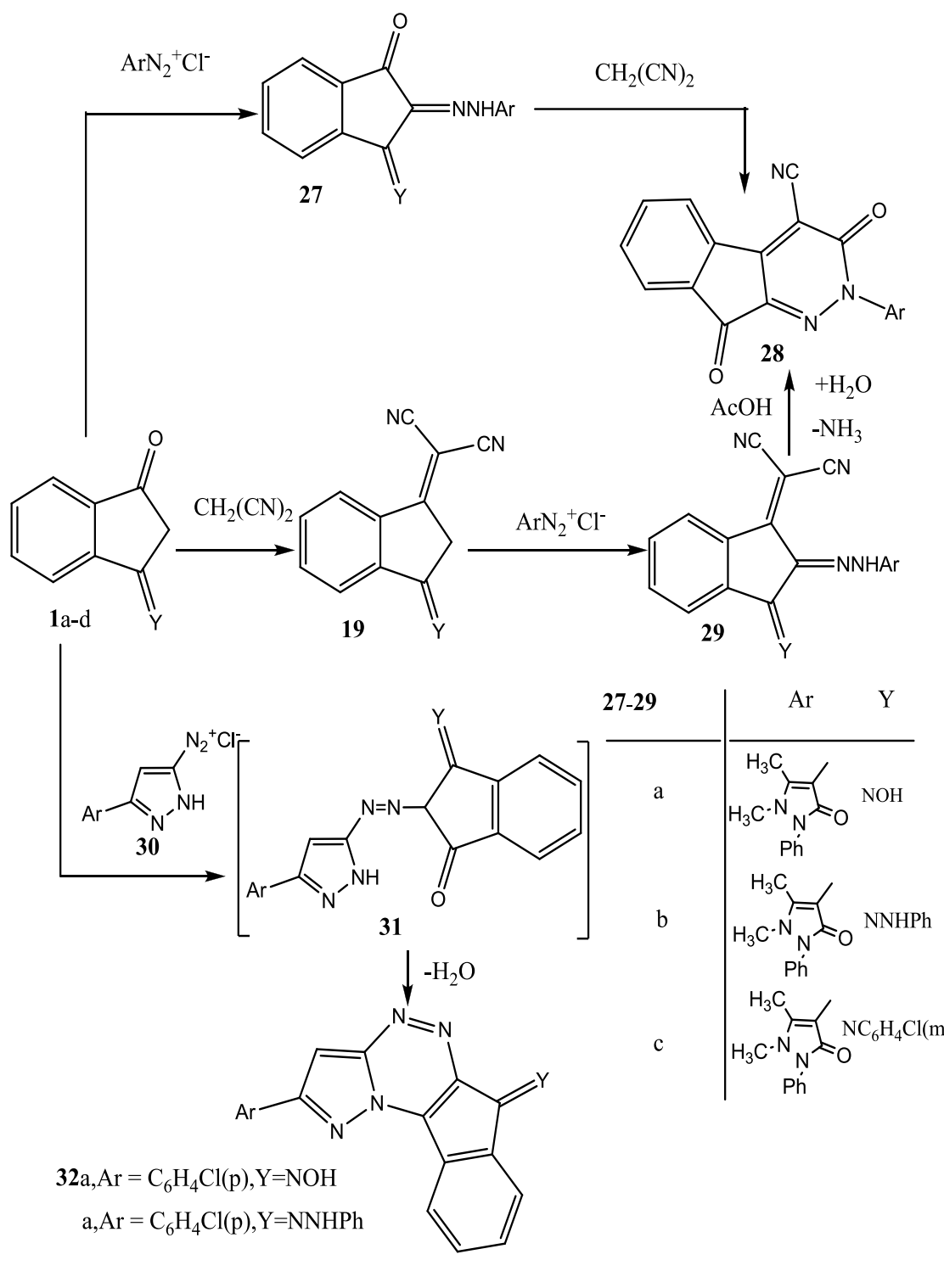

Scheme 5

\section{Experimental}

All melting points are uncorrected and have been measured on a Griffin \& George MBF 010T (London) apparatus. Recorded yields correspond to the pure products. IR $(\mathrm{KBr})$ spectra were recorded on a Perkin Elmer SP-880 spectrometer

Egypt. J. Chem. 55, No.2 (2012) 
and ${ }^{1} \mathrm{H}-\mathrm{NMR}$ spectra were measured on a Varian $270 \mathrm{MHz}$ spectrometer in DMSO- $\mathrm{d}_{6}$ as solvent and using TMS as an internal standard. Chemical shifts are reported in $\delta$ units (ppm). Microanalyses were performed on a LECO CHN-932 elemental analyzer and carried out in the Microanalytical Data Unit at Cairo and Mansoura Universities.Mass spectra were recorded on a MS 30(AEI) instrument at $70 \mathrm{eV}$ ionization energy .

General procedure for preparation of indeno[1,2-b]pyridines (7a- $f$ )

A solution of $1 \mathrm{~b}, \mathrm{~d}^{(9)}(0.01 \mathrm{~mol})$ in ethanol $(50 \mathrm{ml})$ was treated with the arylmethylenenitriles $2(0.01 \mathrm{~mol})$ and few drops of piperidine. The reaction mixture was refluxed for $6 \mathrm{hr}$, then left to cool at room temperature. The solids formed were collected by filtration, crystallized from the proper solvent and then identified as $7 \mathrm{a}-\mathrm{f}$.

2-Amino-1-hydroxy-5-oxo-4-phenyl-4,5-dihydro-1H-indeno [1,2-b]pyridine3- carbonitrile (7a)

Brown crystals, from ethanol /1,4-dioxan, m.p. $240-242^{\circ} \mathrm{C}$, yield $70 \%$.$\operatorname{IR}\left(\tilde{v} / \mathrm{cm}^{-1}\right): 3240,3140\left(\mathrm{OH}, \mathrm{NH}_{2}\right), 2220$ (conjugated $\left.\mathrm{CN}\right), 1715(\mathrm{CO}) .{ }^{1} \mathrm{H}-$ NMR $\left(\right.$ DMSO-d $\left._{6}\right)(\delta, p p m): 4.32(\mathrm{~s}, 1 \mathrm{H}, 4-\mathrm{H}), 7.71-8.66(\mathrm{~m}, 9 \mathrm{H}$, aryl H), 8.77 (s, $\left.2 \mathrm{H}, \mathrm{NH}_{2}\right), 11.99$ (s, 1H, OH). $-\mathrm{C}_{19} \mathrm{H}_{13} \mathrm{~N}_{3} \mathrm{O}_{2}$ (315.31) Calcd . C $72.37 \mathrm{H} 4.15 \mathrm{~N}$ 13. Found C $72.24 \mathrm{H} 4.43 \mathrm{~N}$ 13.56. MS : $\mathrm{m} / \mathrm{z}=315$.

2-Amino-1-hydroxy-4-(4'-chlorophenyl)-5-oxo-4,5-dihydro-1H-indeno[1,2-b] pyridine-3-carbonitrile ( $7 b)$

Yellow crystals, from ethanol /DMF, m.p. $242-244^{\circ} \mathrm{C}$, yield $65 \% .-\mathrm{IR}\left(\tilde{v} / / \mathrm{cm}^{-1}\right)$ : 3350, 3263,3148 $\left(\mathrm{OH}, \mathrm{NH}_{2}\right)$, 2214 (conjugated $\left.\mathrm{CN}\right), 1713(\mathrm{CO})$. ${ }^{1} \mathrm{H}-\mathrm{NMR}$ $\left(\mathrm{DMSO}_{6}\right)(\delta, \mathrm{ppm}): 4.28(\mathrm{~s}, 1 \mathrm{H}, 4-\mathrm{H}), 7.71-8.66(\mathrm{~m}, 8 \mathrm{H}, \operatorname{aryl} \mathrm{H}), 8.88\left(\mathrm{~s}, 2 \mathrm{H}, \mathrm{NH}_{2}\right)$, $11.99(\mathrm{~s}, 1 \mathrm{H}, \mathrm{OH}) .-\mathrm{C}_{19} \mathrm{H}_{12} \mathrm{ClN}_{3} \mathrm{O}_{2}$ (349.77) Calcd. C $65.24 \mathrm{H} 3.45 \mathrm{~N} 12.01$ Found C 65.34 H 3.65 N 12.23. MS: $m / z=349$.

2-Amino-4-(4'-bromophenyl)-5-oxo-1-(3'-chlorophenyl)-4,5-dihydro-1H-indeno [1,2-b]pyridine-3-carbonitrile (7c)

Deep brown crystals, from ethanol /DMF, m.p. $270-272^{\circ} \mathrm{C}$, yield 80\%.$\operatorname{IR}\left(\tilde{v} / \mathrm{cm}^{-1}\right): 3460,3311\left(\mathrm{NH}_{2}\right), 2217$ (conjugated $\left.\mathrm{CN}\right), 1717$ (CO).- ${ }^{1} \mathrm{H}-\mathrm{NMR}$ $\left(\mathrm{DMSO}_{6}\right)(\delta, \mathrm{ppm}): 4.93(\mathrm{~s}, 1 \mathrm{H}, 4-\mathrm{H}), 5.96\left(\mathrm{~s}, 2 \mathrm{H}, \mathrm{NH}_{2}\right), 7.12-8.10(\mathrm{~m}, 12 \mathrm{H}$, aryl H) . $-\mathrm{C}_{25} \mathrm{H}_{15} \mathrm{BrClN}_{3} \mathrm{O}$ (488.76) Calcd. C $61.43 \mathrm{H} 3.09 \mathrm{~N} 8.60$ Found C 61.75 $\mathrm{H} 3.94 \mathrm{~N}$ 8.73. MS : $m / z=488$.

2-Amino-4-(4'-hydroxyphenyl)-5-oxo-1-(3'-chlorophenyl)-4,5-dihydro-1Hindeno[1,2-b]pyridine-3-carbonitrile (7d)

Dark green crystals, from ethanol $/ 1,4$-dioxane, m.p. $214-216^{\circ} \mathrm{C}$, yield $70 \%$.$\operatorname{IR}\left(\tilde{v} / \mathrm{cm}^{-1}\right): 3504,3363\left(\mathrm{OH}, \mathrm{NH}_{2}\right), 2211$ (conjugated $\left.\left.\mathrm{CN}\right), 1716(\mathrm{CO})\right)$ ). ${ }^{1} \mathrm{H}-$ NMR $\left(\right.$ DMSO-d $\left._{6}\right)(\delta, \mathrm{ppm}): 4.93(\mathrm{~s}, 1 \mathrm{H}, 4-\mathrm{H}), 5.88\left(\mathrm{~s}, 2 \mathrm{H}, \mathrm{NH}_{2}\right), 7.12-8.10(\mathrm{~m}$, $12 \mathrm{H}$, aryl $\mathrm{H}), 10.35(\mathrm{~s}, 1 \mathrm{H}, \mathrm{OH})$.- $\mathrm{C}_{25} \mathrm{H}_{16} \mathrm{ClN}_{3} \mathrm{O}_{2}(425.86)$ Calcd. C $70.50 \mathrm{H} 3.78 \mathrm{~N}$ 8.60 Found $\mathrm{C} 70.12 \mathrm{H}, 4.66 \mathrm{~N}$ 8.73. MS : $m / z=425$. 
Ethyl 2-amino-1-hydroxy-5-oxo-4- (4'-hydroxyphenyl)-4,5- dihydro-1H-indeno [1,2-b] pyridine-3-caboxylate $(7 e)$

Brown crystals, from ethanol /1,4-dioxan, m.p.294-296 ${ }^{\circ} \mathrm{C}$, yield $65 \%$.-IR $\left(\tilde{v} / \mathrm{cm}^{-1}\right): 3424,3312\left(\mathrm{OH}, \mathrm{NH}_{2}\right), 1715$ (CO), 1684 (CO ester).- ${ }^{1} \mathrm{H}-\mathrm{NMR}$ (DMSO$\left.\mathrm{d}_{6}\right)(\delta, \mathrm{ppm}): 1.25-131\left(\mathrm{t}, 3 \mathrm{H}, \mathrm{CH}_{3}\right), 4.32-4.52\left(\mathrm{q}, 2 \mathrm{H}, \mathrm{CH}_{2}\right), 4.65(\mathrm{~s}, 1 \mathrm{H}, 4-\mathrm{H}), 6.64(\mathrm{~s}$, $\left.2 \mathrm{H}, \mathrm{NH}_{2}\right), 7.17-7.88\left(\mathrm{~m}, 8 \mathrm{H}\right.$, aryl H), 9.95(s,1H,OH). $-\mathrm{C}_{21} \mathrm{H}_{18} \mathrm{~N}_{2} \mathrm{O}_{5}$ (378.37) Calcd. C 66.65 H 4.79 N 7.40 Found C 66.48 H 5.04 N 7.36. MS : $m / z=378$.

Ethyl 2-amino-1-hydroxy-5- oxo-4-(4'-methoxyphenyl)- 4,5- dihydro-1H-indeno [1,2-b] pyridine-3-caboxylate $(7 f)$

Brown crystals, from ethanol / DMF, m.p. $>300^{\circ} \mathrm{C}$, yield $68 \% .-\operatorname{IR}\left(\tilde{v} / \mathrm{cm}^{-1}\right)$ : 3524, 3312( $\left.\mathrm{OH}, \mathrm{NH}_{2}\right), 1715(\mathrm{CO}), 1684\left(\mathrm{CO}\right.$ ester). $-\mathrm{C}_{22} \mathrm{H}_{18} \mathrm{~N}_{2} \mathrm{O}_{5}$ (390.38) Calcd. C 67.68 H 4.64 N 7.18 Found C 67.72 H 4.46 N 7.24. MS : $m / z=390$.

Preparation of spiro [(2-amino-1,3-disubstituted indeno[1,2-b] pyridine)(5H),3'-[3H]indole]-2',5(1'H)diones (10a-f)

A mixture of $1 \mathrm{~b}-\mathrm{d}(0.01 \mathrm{~mol})$ in ethanol $(50 \mathrm{ml})$ was treated with $(0.01 \mathrm{~mol})$ of the ylidenenitriles $8 \mathrm{a}, \mathrm{b}$. The reaction mixture was stirred for $3 \mathrm{hr}$ at room temperature. The formed precipitates were collected by filtration, crystallized from the suitable solvent and then identified as 10a-f.

2-Amino-1-hydroxy-2,5-dioxo-1,5-dihydrospiro[indeno[1,2-b]pyridine-4,3indoline--3-carbonitrile (10a)

Red crystals, from DMF, no melt $<300^{\circ} \mathrm{C}$, yield $60 \%$.-IR $\left(\tilde{\mathrm{v}} / \mathrm{cm}^{-1)}: 3450\right.$, 3388, 3329(OH,NH, $\left.\mathrm{NH}_{2}\right), 2208$ (conjugated $\left.\mathrm{CN}\right) 1699$ ( CO) ,1625(CO amide). $\mathrm{C}_{20} \mathrm{H}_{12} \mathrm{~N}_{4} \mathrm{O}_{3}(356.33)$ Calcd. C 67.40 H $3.39 \mathrm{~N} 15.72$ Found C $67.76 \mathrm{H} 3.25 \mathrm{~N}$ 15.67. MS : $\mathrm{m} / \mathrm{z}=356$.

2-Amino-1-(phenylamino)- 2,5- dioxo-1,5- dihydro spiro [indeno [1,2-b] pyridine-4,3-indoline ]-3-carbonitrile (10b)

Orange crystals, from ethanol/ DMF, m.p. $240-242^{\circ} \mathrm{C}$, yield $62 \% .-\operatorname{IR}\left(\tilde{v} / \mathrm{cm}^{-1}\right)$ : 3490, 3426, 3348, $3242\left(\mathrm{NH}_{2}, \mathrm{NH}\right), 2186$ (conjugated CN) 1704( CO) ,1638(CO amide).- ${ }^{1} \mathrm{H}-\mathrm{NMR}\left(\mathrm{DMSO}-\mathrm{d}_{6}\right)(\delta, \mathrm{ppm}): 6.58\left(\mathrm{~s}, 2 \mathrm{H}, \mathrm{NH}_{2}\right), 7.01-7.67(\mathrm{~m}, 13 \mathrm{H}$, aryl $\mathrm{H}), 9.13,11.23(2 \mathrm{~s}, 2 \mathrm{H}, 2 \mathrm{NH}) .-\mathrm{C}_{26} \mathrm{H}_{17} \mathrm{~N}_{5} \mathrm{O}_{2}$ (431.44) Calcd. C $72.37 \mathrm{H} 3.97 \mathrm{~N}$ 16.23 Found C 72.70 H 4.23 N 16.43. MS: $m / z=431$.

2-Amino-1-(3-chloropheny)-2,5-dioxo-1,5-dihydrospiro [indeno[1,2-b] pyridine4,3-indoline]-3-carbonitrile (10c)

Faint brown crystals, from DMF, m.p. $250-252^{\circ} \mathrm{C}$, yield $70 \% .-\operatorname{IR}\left(\tilde{v} / \mathrm{cm}^{-1}\right)$ : 3530, 3462, 3358, $3302\left(\mathrm{NH}_{2}, \mathrm{NH}\right), 2182$ (conjugated $\left.\mathrm{CN}\right) 1716(\mathrm{CO}), 1641$ (CO amide).- $\mathrm{C}_{26} \mathrm{H}_{15} \mathrm{ClN}_{4} \mathrm{O}_{2}$ (450.87) Calcd . C 69.25 H 3.35 N 12.43 Found C 69.42 H 3.62 N 12.36. MS : $m / z=450$.

Ethyl 2-amino-1-hydroxy-2,5-dioxo-1,5-dihydrospiro [indeno [1,2-b] pyridine4,3-indoline]-3-carboxylate (10d)

Orange crystals, from DMF, no melt $<300{ }^{\circ} \mathrm{C}$, yield $65 \%$.IR $\left(\tilde{v} / \mathrm{cm}^{-1}\right)$ : 3449, 3384, 3271 ( $\left.\mathrm{NH}_{2}, \mathrm{NH}, \mathrm{OH}\right), 1704$ (CO), 1670 (CO ester), 1646(CO amide).

Egypt. J. Chem. 55, No.2 (2012) 
$-{ }^{1} \mathrm{H}-\mathrm{NMR}\left(\mathrm{DMSO}^{-} \mathrm{d}_{6}\right)(\delta, \mathrm{ppm}): 1.32-1.39\left(\mathrm{t}, 3 \mathrm{H}, \mathrm{CH}_{3}\right), 4.38-4.45\left(\mathrm{q}, 2 \mathrm{H}, \mathrm{CH}_{2}\right)$, 7.15-7.81 (m, 8H, aryl H), $8.76\left(\mathrm{~s}, 2 \mathrm{H}, \mathrm{NH}_{2}\right), 9.15,10.04(2 \mathrm{~s}, 2 \mathrm{H}, \mathrm{NH}$ and $\mathrm{OH})$.$\mathrm{C}_{22} \mathrm{H}_{17} \mathrm{~N}_{3} \mathrm{O}_{5}(403.38)$ Calcd. C $65.50 \mathrm{H} 4.24 \mathrm{~N} 10.42$ Found C $65.65 \mathrm{H} 4.21 \mathrm{~N}$ 10.33. MS : $\mathrm{m} / \mathrm{z}=403$.

Ethyl 2-amino-1-phenylamino-2,5-dioxo-1,5-dihydrospiro [indeno[1,2-b] pyridine4,3-indoline]-3-carboxylate (10e)

Yellow crystals, from ethanol/ DMF, no melt $<300^{\circ} \mathrm{C}$, yield $60 \%$.IR $\left(\tilde{\mathrm{v}} / \mathrm{cm}^{-1}\right): 3515,3448,3394\left(\mathrm{NH}_{2}, \mathrm{NH}\right), 1710(\mathrm{CO}), 1675(\mathrm{CO}$ ester $), 1651(\mathrm{CO}$ amide).- $\mathrm{C}_{28} \mathrm{H}_{22} \mathrm{~N}_{4} \mathrm{O}_{4}(478.49)$ Calcd. C $70.27 \mathrm{H} 4.63 \mathrm{~N} 11.59$ Found $\mathrm{C} 70.35 \mathrm{H}$ $4.42 \mathrm{~N}$ 11.71. MS: $\mathrm{m} / \mathrm{z}=478$.

Ethyl 2-amino-1-(3-chloropheny)-2,5-dioxo-1,5-dihydrospiro [indeno[1,2b]pyridine-4,3-indoline]-3-carboxylate (10f)

Orange crystals, from DMF, m.p. $264-266^{\circ} \mathrm{C}$, yield $63 \% .-\operatorname{IR}\left(\tilde{v} / \mathrm{cm}^{-1}\right): 3468$, 3393 $\left(\mathrm{NH}_{2}, \mathrm{NH}\right), 1706(\mathrm{CO}), 1680$ (CO ester), 1657(CO amide)..- $\mathrm{C}_{28} \mathrm{H}_{20} \mathrm{ClN}_{3} \mathrm{O}_{4}$ (497.92) Calcd. C 67.53 H 4.04 N 8.44 Found C 67.81 H 4.21 N 8.53. MS: $m / z=$ 497 .

Synthesis of indeno[1,2-b]pyridine derivatives (14a-c)

A solution of $1 \mathrm{~b}-\mathrm{d}(0.01 \mathrm{~mol})$ in ethanol $(50 \mathrm{ml})$ containing $(0.1 \mathrm{ml})$ piperidine, was treated with $(0.01 \mathrm{~mol})$ of ethoxymethylenemalononitrile (12). The reaction was heated under reflux for $6 \mathrm{hr}$. The solvent was concentrated to its half volume then left to cool. The precipitates formed were collected by filtration, crystallized from the proper solvent and then identified as (14a-c). (14a)

1-Hydroxy-2-imino-5-oxo-2,5-dihydro-1H-indeno[1,2-b]pyridine-3-carbonitrile

Brown crystals from ethanol, m.p. $240-242^{\circ} \mathrm{C}$, yield $60 \%$.IR $\left(\tilde{v} / \mathrm{cm}^{-1}\right): 3242$ $(\mathrm{NH}), 2200$ (conjugated $\mathrm{CN}$ ), 1701(CO), $1624(\mathrm{C}=\mathrm{N}) .-\mathrm{C}_{13} \mathrm{H}_{7} \mathrm{~N}_{3} \mathrm{O}_{2}$ (237.22) Calcd. C 65.82 H 2.97 N 17.71 Found C 65.79 H 3.03 N 17.65. MS : $m / z=237$.

2-Imino-5-oxo-1- (phenylamnio)-2,5- dihydro-1H-indeno [1,2-b] pyridine-3carbonitrile (14b)

Deep green crystals from 1,4-dioxane, m.p. $290-292^{\circ} \mathrm{C}$, yield $63 \%$-IR( $\left(\tilde{v} / \mathrm{cm}^{1}\right): 3395(\mathrm{NH}), 2199\left(\right.$ conjugatedCN), $1697(\mathrm{CO}) .{ }^{1} \mathrm{H}-\mathrm{NMR}$ (DMSO-d ${ }_{6}$ ) $(\delta, \mathrm{ppm}): 7.53-7.95(\mathrm{~m}, 10 \mathrm{H}$, aryl $\mathrm{H}), 8.4-8.8(2 \mathrm{~s}, 2 \mathrm{H}, 2 \mathrm{NH}) .-\mathrm{C}_{19} \mathrm{H}_{12} \mathrm{~N}_{4} \mathrm{O}(312.32)$ Calcd. C 73.06 H 3.87 N 17.94 Found C 73.21 H 4.16 N 18.03. MS : $\mathrm{m} / z=312$.

1-[(3'-Chlorophenyl) amino]-2- imino-5-oxo-2,5- dihydro-1H-indeno[1,2-b] pyridine-3-carbonitrile (14c)

Green crystals from ethanol/DMF, m.p. $140-142^{\circ} \mathrm{C}$, yield $60 \%$. $-\operatorname{IR}\left(\tilde{v} / \mathrm{cm}^{-1}\right)$ : $3450(\mathrm{NH}), 2201$ (conjugated $\mathrm{CN}$ ), $1718(\mathrm{CO}) \cdot-\mathrm{C}_{19} \mathrm{H}_{10} \mathrm{ClN}_{3} \mathrm{O}$ (331.75) Calcd.C 68.78 H 3.03 N 12.67 Found C 68.53 H 3.91 N 12.56. MS : $m / z=331$. 
Synthesis of 4H-indeno[1,2-b]pyrans (17a-c)

Method A

A suspension of 2-(arylmethylene)indane-1,3-dione 15 (0.01mole) in ethanol (50 $\mathrm{ml})$ containing piperidine $(0.5 \mathrm{ml})$ was treated with 2-cyano- $\mathrm{N}-(4-$ phenyl-2-thioxo$3(2 \mathrm{H})$-thiazolyl ) acetamide $3 \mathrm{c}(0.01$ mole $)$. The reaction mixture was refluxed for $6 \mathrm{hr}$. The solid products obtained were crystallized and identified as $17 \mathrm{a}-\mathrm{c}$.

\section{Method B}

Refluxing of indan-1,3-dione (1a) $(0.01 \mathrm{~mol})$ and $18(0.01 \mathrm{~mol})$ using the same previous procedure afforded $17 \mathrm{a}-\mathrm{c}$.

2-Amino-5-oxo-4- phenyl-N- (4-phenyl-2-thioxo-3(2H)- thiazolyl-4H-indeno [1,2-b] pyran-3-carboxamide (17a)

Red crystals from ethanol/DMF, m.p. $>300{ }^{\circ} \mathrm{C}$, yield $60 \% .-\operatorname{IR}\left(\tilde{v} / \mathrm{cm}^{-1}\right): 3450$, 3363( $\left.\mathrm{NH}_{2}, \mathrm{NH}\right), 1705(\mathrm{CO}), 1679\left(\mathrm{CO}\right.$ amide) $-\mathrm{C}_{28} \mathrm{H}_{19} \mathrm{~N}_{3} \mathrm{O}_{3} \mathrm{~S}_{2}$ (509.58) Calcd.C $66.00 \mathrm{H} 3.75 \mathrm{~N} 8.25$ Found C 66.11 H 4.03 N 8.13. MS : $m / z=509$.

2-Amino-5-oxo-4- (4-methoxyphenyl)-N-(4-phenyl-2-thioxo-3 (2H)-thiazolyl4H-indeno[1,2-b]pyran-3-carboxamide (17b)

Deep red crystals from ethanol, m.p. $175-177^{\circ} \mathrm{C}$, yield $63 \% .-\operatorname{IR}\left(\tilde{v} / \mathrm{cm}^{-1}\right): 3500$, 3487, $3380\left(\mathrm{NH}_{2}, \mathrm{NH}\right), 1707(\mathrm{CO}), 1660$ (COamide).- ${ }^{1} \mathrm{H}-\mathrm{NMR}$ (DMSO-d $\left.{ }_{6}\right)(\delta$, ppm): 3.83(s,3H, $\left.\mathrm{OCH}_{3}\right), \quad 5.23(\mathrm{~s}, 1 \mathrm{H}, \mathrm{pyranH}-4), 7.26\left(\mathrm{~s}, 2 \mathrm{H}, \mathrm{NH}_{2}\right), 6.62(\mathrm{~s}, 1 \mathrm{H}$, thiazole $\mathrm{H}-5), 7.40-7.76\left(\mathrm{~m}, 13 \mathrm{H}\right.$, aryl H), 9.86(s, 1H,NH) . $\mathrm{C}_{29} \mathrm{H}_{21} \mathrm{~N}_{3} \mathrm{O}_{4} \mathrm{~S}_{2}(539.61)$ Calcd. C 64.45 H 3.92 N 7.79 Found C 64.73 H 3.87 N 7.83. MS : $m / z=539$.

2-Amino4-(4-cholorophenyl)- 5-oxo- N-(4-phenyl-2-thioxo-3 (2H)-thiazolyl4H-indeno[1,2-b]pyran-3-carboxamide $(17 c)$

Deep red crystals from ethanol, m.p. $290-292^{\circ} \mathrm{C}$, yield $70 \% .-\mathrm{IR}\left(\tilde{v} / \mathrm{cm}^{-1}\right)$ : 3484, 3395( $\left.\mathrm{NH}_{2}, \mathrm{NH}\right), 1712(\mathrm{CO}), 1665\left(\mathrm{CO}\right.$ amide).- $\mathrm{C}_{28} \mathrm{H}_{18} \mathrm{ClN}_{3} \mathrm{O}_{3} \mathrm{~S}_{2}$ (544.03) Calcd.C 61.81 H 3.33 N 7.72 Found C 61.75 H 3.43 N 7.64. MS: $m / z=455$.

Formation of indanylidenepropanedinitriles (19a-c)

A suspension of $1 \mathrm{~b}-\mathrm{d}(0.01 \mathrm{~mol})$ and $(0.01 \mathrm{~mol})$ of malononitrile in dry benzene $(50 \mathrm{ml})$ containing ammonium acetate $(1 \mathrm{gm})$ and acetic acid $(1 \mathrm{ml})$ was refluxed for $6 \mathrm{hr}$ using water separator. The solvent was concentrated in vacuo and the solid products were filtered off, crystallized from ethanol to give 19a-c.

3-Hydroxyiminoindanylidenepropanedinitrile (19a)

Brown crystals from ethanol / DMF, m.p.>300 ${ }^{\circ} \mathrm{C}$, yield $70 \%$. $-\operatorname{IR}\left(\tilde{v} / \mathrm{cm}^{-1}\right)$ : 3250, 3160(OH), 2205(conjugated $\mathrm{CN}), 1630(\mathrm{C}=\mathrm{N}) .-\mathrm{C}_{12} \mathrm{H}_{7} \mathrm{~N}_{3} \mathrm{O}$ (209.20) Calcd. C 68.89 H 3.37 N 20.09 Found C 68.74 H 3.50 N 20.05. MS : $m / z=209$.

3-Phenylhydrazonoindanylidenepropanedinitrile (19b)

It was prepared according to the procedure previously reported in the literature $^{(4)}$ 
3-(3'-Chlorophenylimino)indanylidenepropanedinitriles (19c)

Violet crystals from ethanol, m.p. $206-208^{\circ} \mathrm{C}$,yield $75 \% .-\mathrm{IR}\left(\tilde{v} / \mathrm{cm}^{-1}\right)$ : $3450(\mathrm{NH}), 2200$ (conjugated $\mathrm{CN}), 1635(\mathrm{C}=\mathrm{N}) \cdot-\mathrm{C}_{18} \mathrm{H}_{10} \mathrm{ClN}_{3}(303.74) \quad$ Calcd. C 71.18 H 3.32 N 13.83 Found C 71.12 H 3.21 N 13.67. MS : $m / z=303$.

Formation of fluoreneoximes (23a-d and 26)

To a suspension of $19 \mathrm{a}(0.01 \mathrm{~mol})$ in ethanol $(50 \mathrm{ml})$ catalyzed by piperidine $(0.1 \mathrm{ml})$ were added $(0.01 \mathrm{~mol})$ of arylmethylenenitriles 2 or $\beta$-nitrostyrene (24). The reaction mixture was refluxed for $10 \mathrm{hr}$. The crystalline solids, which separated out during reflux, were cooled, filtered, recrystallized from the proper solvent and identified as 23 and 26.

3-Amino-9-(hydroxyimino)-1-phenyl-9H-fluorene-2,4-dicarbonitrile (23a)

Brown crystals from DMF, m.p. $280-282^{\circ} \mathrm{C}$, yield $63 \% .-\operatorname{IR}\left(\tilde{v} / \mathrm{cm}^{-1}\right)$ : 3340 , $3209\left(\mathrm{NH}_{2}, \mathrm{OH}\right), 2191$ (conjugated $\left.\mathrm{CN}\right), 1620(\mathrm{C}=\mathrm{N}) .-\mathrm{C}_{21} \mathrm{H}_{12} \mathrm{~N}_{4} \mathrm{O}(336.35)$ Calcd. C 74.99 H 3.60 N 16.66 Found C 75.12 H 3.52 N 16.72. MS : $m / z=336$. (23b)

3-Amino-1- (4'-chlorophenyl)-9- (hydroxyimino)- 9H-fluorene-2,4-dicarbonitrile

Brown crystals ethanol/ DMF, m.p. $>300{ }^{\circ} \mathrm{C}$, yield $66 \% .-\operatorname{IR}\left(v_{\max }, \mathrm{cm}^{-1}\right): 3460$, $3319\left(\mathrm{NH}_{2}, \mathrm{OH}\right), 2193$ (conjugated $\left.\mathrm{CN}\right), 1660(\mathrm{C}=\mathrm{N}) \cdot-\mathrm{C}_{21} \mathrm{H}_{11} \mathrm{ClN}_{4} \mathrm{O}(370.80)$ Calcd. C 68.02 H 2.99 N 15.11 Found C 68.11 H 3.12 N 15.08. MS : $m / z=370$.

Ethyl 3-amino-1-(4'-chlorophenyl)-4-cyano-9-(hydroxyimino)-9H-fluorene-2carboxylate $(23 c)$

Deep brown crystals ethanol/ DMF, m.p. $>300{ }^{\circ} \mathrm{C}$, yield $70 \% .-\operatorname{IR}\left(\tilde{v} / \mathrm{cm}^{-1}\right)$ : 3337, $3200\left(\mathrm{NH}_{2}, \mathrm{OH}\right), 2203$ (conjugated $\left.\mathrm{CN}\right), 1670(\mathrm{CO}), 1604(\mathrm{C}=\mathrm{N})$.$\mathrm{C}_{23} \mathrm{H}_{16} \mathrm{ClN}_{3} \mathrm{O}_{3}$ (417.85) Calcd. C 66.11 H 3.86 N 10.06 Found C $66.00 \mathrm{H} 4.11 \mathrm{~N}$ 10.23. MS : $\mathrm{m} / z=417$.

Ethyl 3-amino-4-cyano-9-(hydroxyimino)-1-(4-hydroxyphenyl)-9H-fluorene2-carboxylate (23d)

Brown crystals ethanol / DMF, no melt $<300{ }^{\circ} \mathrm{C}$, yield $65 \%$. $-\operatorname{IR}\left(\tilde{v} / \mathrm{cm}^{-1}\right)$ : 3490, 3378, 3209( $\left.\mathrm{NH}_{2}, \mathrm{OH}\right), 2205($ conjugated $\left.\mathrm{CN}), 1660(\mathrm{CO}), 1610(\mathrm{C}=\mathrm{N})\right) .-{ }^{1} \mathrm{H}-$ NMR (DMSO-d $\left.{ }_{6}\right)(\delta, p p m): 1.2-1.4\left(\mathrm{t}, 3 \mathrm{H}, \mathrm{CH}_{3}\right), 4.2-4.4\left(\mathrm{q}, 2 \mathrm{H}, \mathrm{CH}_{2}\right), 6.8(\mathrm{~s}$, $\left.2 \mathrm{H}, \mathrm{NH}_{2}\right), 7.2-7.95(\mathrm{~m}, 8 \mathrm{H}$, aryl $\mathrm{H}), 8.8,9.6(2 \mathrm{~s}, 2 \mathrm{H}, 2 \mathrm{OH}) \cdot \mathrm{C}_{23} \mathrm{H}_{17} \mathrm{~N}_{3} \mathrm{O}_{4}(399.41)$ Calcd.C 69.17 H 4.29 N 10.52 Found C 69.19 H 4.12 N 10.67. MS : $m / z=399$.

3-Amino-9-(hydroxyimino)-2-nitro-1-phenyl-9H-fluorene-4-carbonitrile (26)

Brown crystals ethanol, m.p. $278-279^{\circ} \mathrm{C}$, yield 70\%.-IR $\left(\tilde{v} / \mathrm{cm}^{-1}\right): 3333,3194$, $\left(\mathrm{NH}_{2}, \mathrm{OH}\right), 2199$ (conjugated $\left.\left.\mathrm{CN}\right), 1630(\mathrm{C}=\mathrm{N})\right), 1365\left(\mathrm{NO}_{2}\right) \cdot-\mathrm{C}_{20} \mathrm{H}_{12} \mathrm{~N}_{4} \mathrm{O}_{3}(356.33)$ Calcd.C 67.41 H 3.39 N 15.72 Found C 67.33 H 3.52 N 15.68. MS : $m / z=356$.

Preparation of 2-arylhydrazonoindan-1-one derivatives (27a-c)

A cold solution of $1 \mathrm{~b}-\mathrm{d}(0.01 \mathrm{~mol})$ in ethanol $(100 \mathrm{ml})$ was treated with a saturated sodium acetate solution $(10 \mathrm{ml})$ and then with the aryldiazonium 
chloride. The mixture was left in the refrigerator for $24 \mathrm{hr}$. The solid products were collected by filtration, crystallized from ethanol to give $27 \mathrm{a}-\mathrm{c}$.

4-(2-(1-Hydroxyimino)-3-1H-inden-2-(3H)-ylidene)hyrazinyl)-1,5-dimethyl2-phenyl-1H-pyrazol-3(2H)-one( 27a)

Orange crystals, m.p. $180^{\circ} \mathrm{C}$, yield $70 \%$. -IR $\left(\tilde{v} / \mathrm{cm}^{-1}\right): 3490,3450,3370(\mathrm{OH}$, $\mathrm{NH}), 1706(\mathrm{CO}), 1655$ (CO antipyrinyl), 1630(C=N).-C20H17N5O3 (375.39) Calcd. C 63.99 H 4.56 N 18.66 Found C 64.03 H 4.43 N 18.53. MS : $m / z=375$.

1,5-Dimethyl- 4(2- (1-oxo-3-(2- phenylhyrazono)-1H-inden-2(3H)-ylidene) hyrazinyl-2-phenyl-1H-pyrazol-3-(2H)-one (27b)

Yellow crystals, m.p. $160-162^{\circ} \mathrm{C}$, yield $73 \%$. - IR( $\left(\tilde{\mathrm{v}} / \mathrm{cm}^{-1}\right)$ : $3435,3340(\mathrm{NH})$, 1714 (CO), 1645 (CO antipyrinyl), $1620(\mathrm{C}=\mathrm{N})$.-1H-NMR: Insoluble.C26H22N6O2 (450.50) Calcd. C 69.32 H 4.92 N 18.65 Found C 69.43 H 4.85 N 18.73. MS : $m / z=450$.

4-(2-((E)-1-(3-chlorophenylimino)-3-oxo-1H-inden-2(3H)-ylidene) hydrazinyl)1,5-dimethyl-2-phenyl-1H-pyrazol-3(2H)-one (27c)

Orange crystals, m.p. $169-170^{\circ} \mathrm{C}$, yield $63 \% . \operatorname{IR}\left(\tilde{\mathrm{v}} / \mathrm{cm}^{-1}\right)$ : $3485,(\mathrm{NH})$, 1716(CO), 1647(CO antipyrinyl), 1620(C=N).-C26H20ClN5O2 (469.93) Calcd. C 66.45 H 4.29 N 14.90 Found C 66.61 H 4.35 N 14.88. MS : $m / z=469$.

Preparation of indeno[2,1-c]pyridazines (28a-c)

Method A

A mixture of $27(0.01 \mathrm{~mol})$ and malononitrile $(0.01 \mathrm{~mol})$ in dry benzene $(100$ $\mathrm{ml}$ ) was refluxed for $3 \mathrm{hr}$ in presence of ammonium acetate $(1 \mathrm{gm})$ and acetic acid (1ml) using Dean- Stark trap. The solvent was concentrated to its half volume and left to cool. The solids deposited were collected by filtration, crystallized from ethanol/DMF to give 28a-c .

\section{Method B}

Compounds $29(0.01 \mathrm{~mol})$ in glacial acetic acid $(30 \mathrm{ml})$ were refluxed for $5 \mathrm{hr}$. The solvent was removed in vacuo and the formed solid products were crystallized and identified as 28 .

2-(1,5-Dimethyl-3-oxo-2-phenyl-2,3-dihydro-1H-pyrazol-4-yl)-9-(hydroxyimino)3-oxo-3,9-dihydro-2H-indeno[2,1-c] pyridazine-4-carbonitrile (28a)

Brown crystals, no melt $<300^{\circ} \mathrm{C}$, yield $62 \%$. $-\mathrm{IR}\left(\tilde{\mathrm{v}} / \mathrm{cm}^{-1}\right): 3440(\mathrm{OH}), 2203$ (conjugated $\mathrm{CN}), \quad 1670(\mathrm{CO}), \quad 1645(\mathrm{CO}$ antipyrinyl) .-1H-NMR(DMSO-d6)

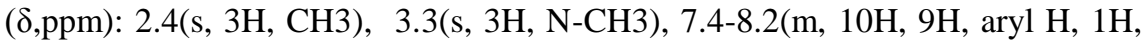
$\mathrm{OH})$-C23H16N6O3 (424.42) Calcd.C 65.09 H 3.80 N 19.80 Found C 65.12 H $3.72 \mathrm{~N} 19.93$. MS $: m / z=424$.

2-(1,5-Dimethyl-3-oxo-2-phenyl-2,3-dihydro-1H-pyrazol-4-yl)-9(phenylhydrazone)3-oxo-3,9-dihydro-2H-indeno[2,1-c] pyridazine-4-carbonitrile (28b)

Brown crystals, no melt $<300^{\circ} \mathrm{C}$, yield $63 \% .-\operatorname{IR}\left(\tilde{\mathrm{v}} / \mathrm{cm}^{-1}\right): 3444$ (NH), 2209 (conjugated CN), 1667(CO), 1647(CO antipyrinyl), 1627(C=N).-C29H21N7O2 
(499.54) Calcd. C 69.73 H 4.24 N 19.63 Found C 69.65 H 4.31 N 19.56. MS: $\mathrm{m} / \mathrm{z}=499$.

2-(1,5- Dimethyl- 3-oxo-2- phenyl-2,3- dihydro-1H- pyrazol-4-yl)-9-(N-3chlorophenyl)-3-oxo-3,9-dihydro-2H-indeno[2,1-c] pyridazine-4-carbonitrile(28c)

Deep brown crystals, no melt $<300^{\circ} \mathrm{C}$, yield $60 \%$. IR $\left(\tilde{\mathrm{v}} / \mathrm{cm}^{-1}\right): 2197$ (conjugated $\mathrm{CN}), 1668$ (CO), 1650 (CO antipyrinyl), $1620 \quad(\mathrm{C}=\mathrm{N})$. C29H19ClN6O2 (518.97) Calcd. C 67.12 H 3.69 N 16.19 Found C 67.45 H $3.77 \mathrm{~N} 16.24$. MS: $m / z=518$.

Formation of arylhydrazones (29)

Reaction of aryldiazonium chloride with $19 \mathrm{a}-\mathrm{c}$ using the procedure for preparation of 27 yielded 29 after crystallization from ethanol/DMF .

2-(2-(2- (1,5-Dimethyl-3-oxo-2-phenyl-2,3-dihydro-1H-pyrazol-4-yl)hydrazono)3-(hydrpxyimino)-2,3-dihydro-1H-indene-1-ylidene)malononitrile (29a)

Brown crystals, m.p. $<300^{\circ} \mathrm{C}$, yield $70 \% \cdot-\operatorname{IR}\left(v_{\max }, \mathrm{cm}^{-1}\right): 3323,3201$, (NH,OH), 2218, 2189 (two conjugated $\mathrm{CN}$ ), 1646(CO phenazonyl).- $\mathrm{C}_{23} \mathrm{H}_{17} \mathrm{~N}_{7} \mathrm{O}_{2}$ (423.44) Calcd. C 65.24 H 4.05 N 23.16 Found C 65.33 H 4.12 N 23.24. MS: $\mathrm{m} / \mathrm{z}=423$.

2-(2-(2-(1,5-Dimethyl-3-oxo-2- phenyl-2,3- dihydro-1H-pyrazol-4-yl)hydrazono)3-(2-phenylhydrazono)-2,3-dihydro-1H-indene-1-ylidene)malononitrile (29b)

Deep brown crystals ,m.p. $208-210^{\circ} \mathrm{C}$, yield $75 \% . \operatorname{IR}\left(\tilde{v} / \mathrm{cm}^{-1}\right): 3447,3298$, $(\mathrm{NH}), 2222,2188$ (two conjugated $\mathrm{CN}), 1643(\mathrm{CO}$ phenazonyl), 1630(C=N). $-\mathrm{C}_{29} \mathrm{H}_{22} \mathrm{~N}_{8} \mathrm{O}(498.55)$ Calcd. C $69.87 \mathrm{H} 4.45 \mathrm{~N} 22.48$ Found $\mathrm{C} 69.76 \mathrm{H} 4.11 \mathrm{~N}$ 22.34. MS: $m / z=498$.

2-((3E)-3-(3-chlorophenylimino)-2-(2-(1,5-dimethyl-3-oxo-2-phenyl-2,3-dihydro1H-pyrazol-4-yl)hydrazono)-2,3-dihydro-1H-indene-1-ylidene)malononitrile (29c)

Dark brown crystals, m.p. $160-162^{\circ} \mathrm{C}$ (, yield $60 \% .-\mathrm{IR}\left(\tilde{v} / \mathrm{cm}^{-1}\right): 3443$, $(\mathrm{NH})$, 2220, 2213(two conjugated $\mathrm{CN}$ ), 1647 (CO phenazonyl). $-\mathrm{C}_{29} \mathrm{H}_{20} \mathrm{ClN}_{7} \mathrm{O}$ (517.98) Calcd. C 67.25 H 3.89 N 18.93 Found C 67.13 H 4.12 N 18.86. MS : $m / z=517$.

Formation of 2-[4'-chlorophenyl]pyrazolo[3,2-c]indeno[1,2-e] [1,2,4] triazine derivatives $(32 a, b)$

To a cold solution of $1 \mathrm{~b}, \mathrm{c}(0.01 \mathrm{~mol})$ in ethanol $(50 \mathrm{ml})$ containing saturated sodium acetate solution $(10 \mathrm{ml})$, the diazonium salt 30 prepared from the amine hydrochloride $(0.01 \mathrm{~mol})$ and the equivalent amount of sodium nitrite was added dropwise with stirring. The reaction mixture was left in the refrigerator overnight. The resulting solids were collected by filtration, crystallized from ethanol to give 32a,b .

2-(4'-Chlorophenyl)-6H-indeno[1,2-e]pyrazolo[5,1-c][1,2,4] triazin-6-one oxime (32a)

Orange crystals, m.p. $280-282^{\circ} \mathrm{C}$, yield $86 \% \cdot-\operatorname{IR}\left(\tilde{v} / \mathrm{cm}^{-1}\right): 3300,3132$ $(\mathrm{OH}), 1630(\mathrm{C}=\mathrm{N}) .-\mathrm{C}_{18} \mathrm{H}_{10} \mathrm{ClN}_{5} \mathrm{O}$ (347.77) Calcd. C 62.17 H $2.90 \mathrm{~N} 20.14$ Found C 62.33 H 3.03 N 20.21. MS: $m / z=347$.

Egypt. J. Chem. 55, No.2 (2012) 
2-(4'-Chlorophenyl)- 6H-indeno [1,2-e] pyrazolo [5,1-c][1,2,4] triazin-6-one phenylhydrazone $(32 b)$

Red crystals, m.p. $160-162^{\circ} \mathrm{C}$, yield $75 \% .-\operatorname{IR}\left(\tilde{v} / \mathrm{cm}^{-1}\right): 3249 \quad(\mathrm{NH}), 1673$ $(\mathrm{C}=\mathrm{N}) .-\mathrm{C}_{24} \mathrm{H}_{15} \mathrm{ClN}_{6}(422.88)$ Calcd. C 68.17 H $3.58 \mathrm{~N} 19.87$ Found C $68.34 \mathrm{H}$ $3.43 \mathrm{~N}$ 19.67. MS : $m / z=422$.

\section{References}

1. Verma , R.V., Ila, H. and Singh, M.S., Heteroaromatic annulations studies 2[bis(methylthio)methylene]-1,3-indanedione: Efficient routes to indenofused heterocycles. Tetrahedron, 66, 7389 (2010)

2. El-Zohry, M.F., Elossaily, Y.A., Mohamed, T.A. and Hussein, E.M., Synthesis and reactions of some new spiroindeno[1,2-b]pyran-4,3-indolines. Heterocycles, 75 (4), 955 (2008).

3. El-Taweel, F.M., Osman, A.I., El-Awad, S.M.H. and Elagamey, A.A., New routes for the synthesis of indene derivatives using 1,3-indanedione. Alex. J. Pharm. Sci. 12 (1), 44 (1998)

4. El-Taweel, F.M., Sofan, M.A., Abu El-Maati, T.M. and El-Agamey, A.A., Synthetic routes to fluorenone, indenopyridine, $4 H$-naphtho[2,1-b]pyrans and pyridine derivatives. Bull. Chem. Farm. 140, 306 (2001).

5. El-Taweel, F.M., Sofan, M.A., Ayaad, S.N., Abu El-Maati, T.M. and El-Agamey, A.A., Synthetic routes to indenothiophene, indenopyran , and fluorenone derivatives. Bull. Chem. Farm. 137, 448 (1998).

6. Wang, S., Ma, N., Zhang, G., Shi, F., Jiang, B., Lu, H., Gao, Y. and Tu, S., An efficient and clean synthesis of inedno[1,2-b]pyrazolo4,3-e] pyridine-5(1H)-one derivatives under microwave irradiation in water. J. Heterocycl. Chem. 47, 1283 (2010).

7. Ghahremanzadeh, R., Fereshtehnejad, F. and Bozgir, A., One - pot synthesis of spiro[diindeno[1,2-b:2', 1'-e]pyridine-11,3'-indoline]-triones. J. Heterocycl. Chem. 47, $1031(2010)$

8. Boominathan, M., Nagaraj, M., Muthusubramanian, S. and Krishnakumar, R.V., Efficient atom economical one-pot multicomponent synthesis of densely functionalized $4 H$-chromene derivatives. Tetrahedron, 67, 6057 (2011).

9. Abu El Maati, T.M., Said, S.B., Abu Elenein, N.S., Khodier, M.N. and Sofan, M. A., 3-(Phenylhydrazono)- indan-1-one and 2-dimethylaminomethylene-3-(phenylhydrazono)- indan-1-one as useful synthons for the constraction of new heterocyclic systems . J. Heterocycl . Chem. 40, 481 (2003) .

10. Durden, J.A. and Sousa, A.A., Substituted phenylindanediones as miticides. U.S.Pat. 3, 954, 998 (1976) C.A. 85, 73467x (1976).

11. Afsa, E.M., Etmam,H.A., Hamama, W.S. and Ahmed, A.F.S., Condensation of 2 arylidene-1,3-indanediones with antipyrine and indole. Pharmazie, 50 (8),567 (1995).

Egypt. J. Chem. 55, No.2 (2012) 
12. El-Taweel, F.M., Elagamey, A.A., El-Kenaawy, A.A. and Waly, M.A., Novel synthesis of thiazole , coumarin , pyridine thiophene and thieno [2,3-b] pyridine derivatives. Phosphorous ,Sulfur, Silicon and Related Elements, 176, 215 (2001).

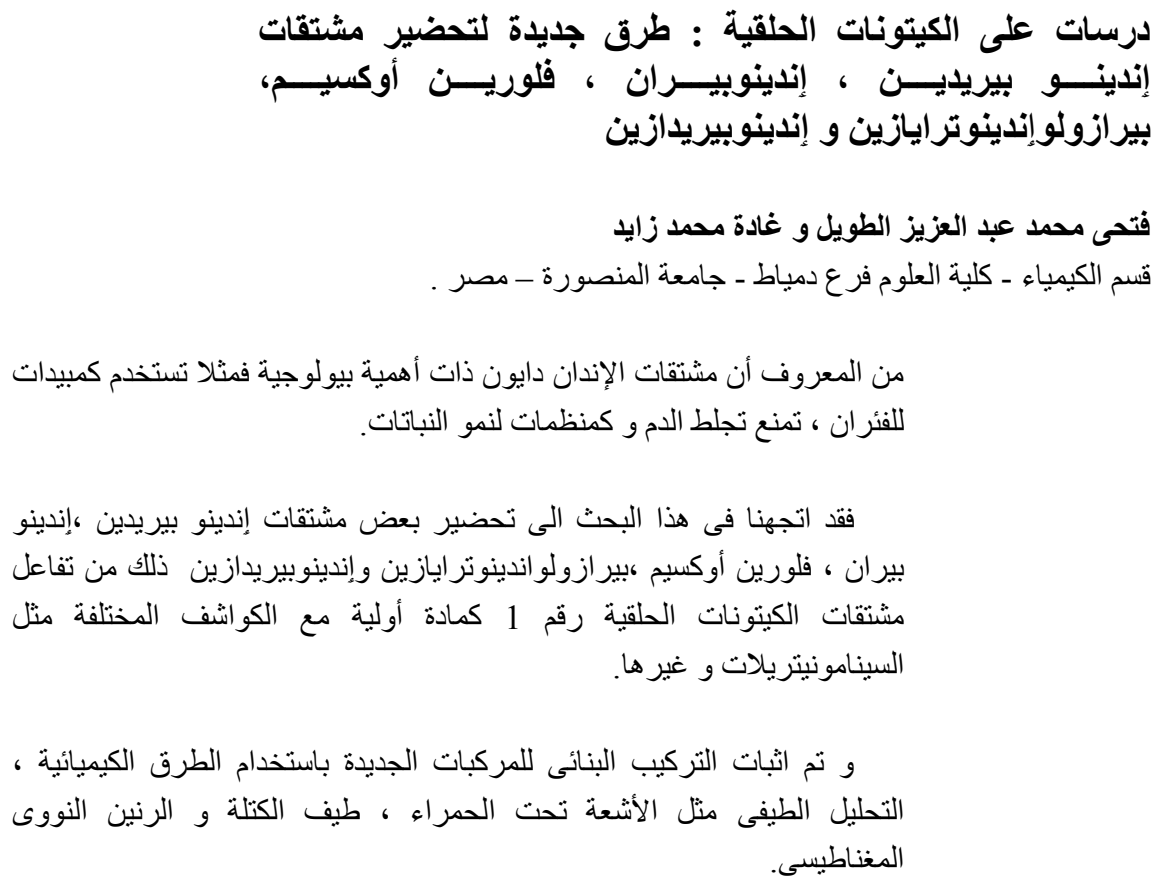

